\title{
Effect of Nanoscale Geometry on Molecular Conformation: Vibrational Sum- Frequency Generation of Alkanethiols on Gold Nanoparticles
}

Champika Weeraman, Achani K. Yatawara, Andrey N. Bordenyuk, and Alexander V. Benderskii*

\author{
Department of Chemistry, Wayne State University, Detroit, MI 48202. \\ RECEIVED DATE (automatically inserted by publisher); E-mail: alex@chem.wayne.edu
}

\section{Supporting Information}

\section{Sample Preparation}

Dodecanethiol capped gold nanoparticles of four different sizes: (1) mean diameter $\langle d\rangle=1.8 \mathrm{~nm}$ with standard deviation $\sigma_{\mathrm{d}}=1.3 \mathrm{~nm}$; (2) $\langle\mathrm{d}\rangle=2.9 \mathrm{~nm} \sigma_{\mathrm{d}}=0.6 \mathrm{~nm}$; (3) $\langle\mathrm{d}\rangle=7.4 \mathrm{~nm} \sigma_{\mathrm{d}}=1.1 \mathrm{~nm}$; and (4) $\langle\mathrm{d}\rangle=23 \mathrm{~nm} \sigma_{\mathrm{d}}=8.1 \mathrm{~nm}$, dissolved in toluene, were purchased from Meliorum Technologies. Spectroscopic studies were done on homogeneously dispersed sub-monolayer nanoparticle samples on an optical grade $\mathrm{CaF}_{2}$ window. Samples were deposited by dropping 10 microliters of gold nanoparticle solution in toluene onto $\mathrm{a} \mathrm{CaF}_{2}$ window (approximate area $2 \mathrm{~cm}^{2}$ ) and allowing the solvent to evaporate by drying in vacuum for 15 hours. Solution concentrations were $3.40 \times 10^{14}$ particles $/ \mathrm{ml}(0.02 \mathrm{mg} / \mathrm{ml})$ for $\langle\mathrm{d}\rangle=1.8 \mathrm{~nm}$ sample, $3.40 \times 10^{14}$ particles $/ \mathrm{ml}(0.08 \mathrm{mg} / \mathrm{ml})$ for $\langle\mathrm{d}\rangle=2.9 \mathrm{~nm}$ sample, $2.44 \times 10^{13}$ particles $/ \mathrm{ml}(0.1 \mathrm{mg} / \mathrm{ml})$ for $\langle\mathrm{d}\rangle=7.4 \mathrm{~nm} \quad$ sample, and $1.24 \times 10^{12}$ particles $/ \mathrm{ml}(0.1 \mathrm{mg} / \mathrm{ml})$ for $\langle\mathrm{d}\rangle=23 \mathrm{~nm}$ sample. Therefore, the expected surface coverage of the nanoparticles is $51.8,51.8,721$, and $14200 \mathrm{~nm}^{2} /$ particle for $1.8 \mathrm{~nm}, 2.9 \mathrm{~nm}, 7.4 \mathrm{~nm}$ and $23 \mathrm{~nm}$ samples, respectively. This corresponds to $5 \%, 14 \%, 7 \%$, and $3 \%$ of a closed-packed (hexagonal)
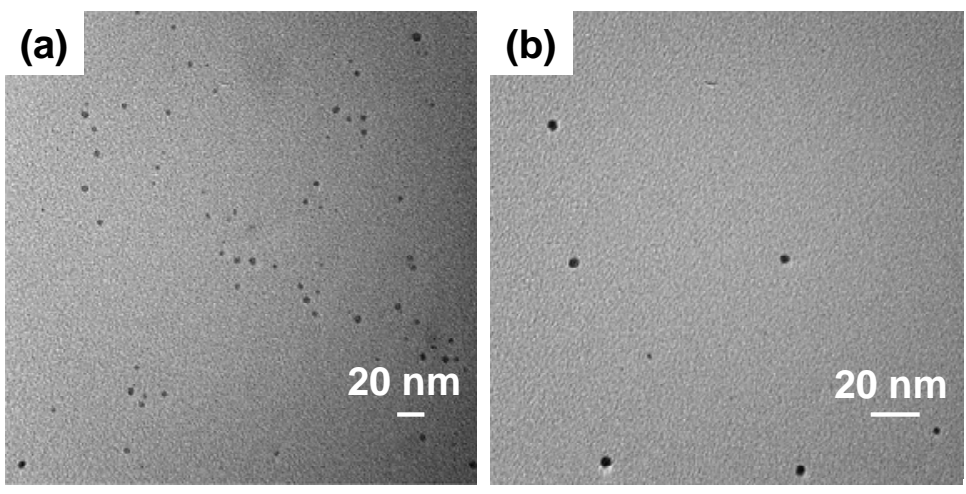
monolayer for the four particle sizes. Transmission electron microscopy (TEM) was performed by depositing samples onto a carbon coated copper grid (mesh 200) under the same conditions as in preparation of VSFG spectroscopic samples (same concentration of drop solution and evaporation conditions). TEM images were recorded by using JELO FasTEM 2010 system operating at $200 \mathrm{kV}$.
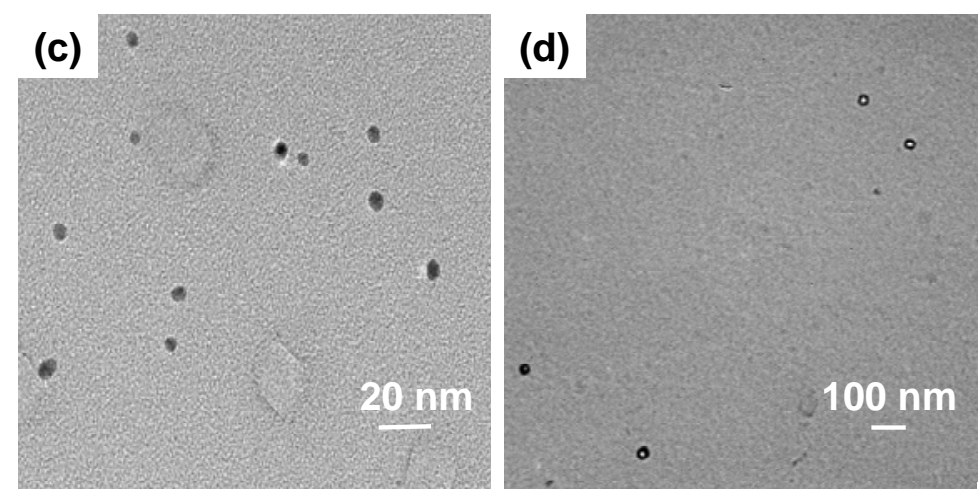

Figure S1. TEM images of drop-deposited Au nanoparticles: (A) $\langle\mathrm{d}\rangle=1.8 \mathrm{~nm}$ Au nanoparticle sample; (B) $\langle\mathrm{d}\rangle=2.9 \mathrm{~nm}$ sample; (C) $\langle d\rangle=7.4 \mathrm{~nm}$ sample; (D) $\langle\mathrm{d}\rangle=23 \mathrm{~nm}$ sample. 


\section{VSFG spectroscopy set-up}

The broad-band vibrational sum frequency generation (VSFG) spectroscopy set-up, which follows the pioneering development by Richter, Petralli-Mallow, and Stephenson [Opt. Lett. 1998, 23, 1594-1596], is based on a high power amplified femtosecond Ti-Sapphire laser system (Spectra Physics Spitfire sub-50 fs HP). $50 \%$ of the $2 \mathrm{~mJ}$ fundamental output pulse ( $800 \mathrm{~nm}$, FWHM $35 \mathrm{fs}$ ) is used to pump an Optical Parametric Amplifier (OPA) followed by the signal-idler difference frequency mixing in a $0.5 \mathrm{~mm}$ thick $\mathrm{AgGaS}_{2}$ crystal producing $75 \mathrm{fs}$ IR pulses $\left(300 \mathrm{~cm}^{-1}\right.$ spectral FWHM) centered at 2900 $\mathrm{cm}^{-1}$. Spectra of the IR pulses were measured using an IR grating (blazed at $5 \mu \mathrm{m}$ ) in the monochromator and liquid nitrogen cooled MCT detector (IR Associates). The broad-band VSFG scheme was employed that uses spectrally broad (FWHM $250 \mathrm{~cm}^{-1}$ ) IR and narrow-band visible pulses to obtain the spectrum by frequency-dispersing the SFG signal. A zero-chirp 4-f design pulse stretcher consisting of a grating, collimating lens and a mirror equipped with a tunable slit was used to narrow the spectrum of the visible (800 nm) pulse from $430 \mathrm{~cm}^{-1}$ of the Spitfire output down to the desired spectral width. Since the full width at half maximum (FWHM) of the spectral features of the nanoparticles samples was determined to be greater than $12 \mathrm{~cm}^{-1}$, we selected the visible pulse spectral width to be $\leq 8 \mathrm{~cm}^{-1}$ Gaussian FWHM. The IR and visible beams were spatially and temporally overlapped at the sample surface with the incidence angle for both beams $65^{\circ}$ from surface normal. The beam diameter at the sample is $\sim 150 \mu \mathrm{m}$ for both visible and IR beams. The laser power at the sample is $4 \mu \mathrm{J} /$ pulse for IR and up to $17-18 \mu \mathrm{J} /$ pulse for the visible at $1 \mathrm{kHz}$ repetition rate. The SFG signal reflected from the sample surface was recollimated, spatially and frequency filtered, passed through a $300 \mathrm{~mm}$ monochromator (Acton Spectra-Pro 300i), and detected using a LN2-cooled CCD (Princeton Instruments Spec-10:100B, 100x1340 pixels). Five pixel binning along the horizontal axis was performed to reduce noise. With this binning, the spectrometer resolution is $2.7 \mathrm{~cm}^{-1}$. The SFG spectrum was obtained by vertical integration of the CCD image. The signals were averaged over many laser shots by using the CCD as the integrator, with acquisition time typically 20-60 min per spectrum. The background spectrum for each spectrum measurement was recorded by blocking IR beam on to the sample for the same acquisition time. The background correction was performed by subtracting from the signal region of interest (e.g., horizontal strips 40-45), a nonilluminated region of background spectrum of the same size. Spikes due to the cosmic X-rays were eliminated using a differential discriminator program. The IR frequencies are calculated by subtracting the central frequency of the narrow visible pulse (measured using the same monochromator) from the SFG frequency. In addition, we calibrate the IR frequency scale using a known SFG surface spectrum of dimethyl sulfoxide (DMSO). We estimate our IR frequency calibration accuracy to be $\pm 2 \mathrm{~cm}^{-1}$. Polarization of the visible beam is controlled by using a zero-order half-wave plate, while the IR beam polarization can be made either horizontal or vertical by using a periscope before the sample. The polarization combination used in these experiments is SSP (SFG-vis-IR). 